\section{Should Key Performance Indicators Be a Component of Performance Assessment for Individual Clinical Pharmacists?}

\section{THE "PRO" SIDE}

Pharmacists represent a scarce human resource in Canadian hospitals, with about 2900 full-time pharmacists providing care for 95000 acute care bed-days in $2010 .^{1,2}$ It is vital to ensure that clinical pharmacists are performing the highest-value activities for the highest-priority patients. Clinical pharmacy leadership and front-line pharmacists require tools to facilitate objective assessment of employee performance, to define benchmarks for performance, and to facilitate the improvement of employee performance.

If we cannot measure something, then we cannot control it. In turn, if we lack the ability to control something, then we cannot improve it. In brief, then, improvement efforts rely on measurement. This concept applies to the performance of both organizations and employees. Clinical pharmacists need objective feedback on their performance to understand what is expected of them and what is required to improve. A process known as performance management can assist with the provision of feedback and the promotion of performance improvement that is aligned with overall organizational performance. ${ }^{3}$ Examples of activities within a performance management system include setting performance targets at the individual level that are linked to organizational objectives, reviewing employees' progress toward those targets, and identifying areas for further development. ${ }^{4}$ The clinical pharmacy key performance indicator (cpKPI) is a tool that facilitates the setting of expectations and the measurement of progress by clinical pharmacists toward identified clinical performance goals. It can be used to quantitatively describe how often an evidence-based process or outcome of care occurs and ultimately to help identify focus areas for future strategies to improve employee performance.

A Canadian collaborative of hospital pharmacists recently developed evidence-informed cpKPIs that aim to advance pharmacy practice and improve patient care. ${ }^{5}$ These cpKPIs will measure a wide variety of activities performed by pharmacists, including performance or review of medication reconciliation on admission; participation in interprofessional patient care rounds; completion of a pharmaceutical care plan; resolution of drug-therapy problems (DTPs); delivery of in-person disease and medication education to inpatients; delivery of in-person discharge education and counselling to inpatients; medication reconciliation on discharge; and bundled, proactive direct patient care activities. ${ }^{5}$ These cpKPIs include both outputs that a pharmacist should achieve, such as DTP resolution, and inputs that should help in achieving the outputs, such as completion of a pharmaceutical care plan. It is advantageous to have a suite of evidence-based cpKPIs, consisting of both input and output activities, to guide performance management because it allows for creation of employee-specific strategies for performance improvement through a focus on both the "how-to" (input) and the final result (output). Using a tool such as the cpKPI may help to standardize performance improvement by defining benchmarks for clinical performance.

The relative value of a clinical pharmacist's outputs, such as DTP resolution, should be determined by the prevalence of the problem, multiplied by the quality of the action, divided by the effort required to manage it. ${ }^{6}$ The quality of the action should be determined from the strength of the supporting evidence for effectiveness, safety, and modifiability of the intervention, and the reliance on pharmacists to perform it. ${ }^{6}$ According to this model, low-value DTP-related examples might include adjusting the dose of folic acid for a critically ill patient who does not have anemia or adding docusate to the medication regimen of a patient who has constipation secondary to opioids. Examples of high-value actions would be initiating an angiotensin-converting enzyme inhibitor for a patient with heart failure or discontinuing an antibiotic for pneumonia at the earliest appropriate time. Developing a list of high-value DTP-related actions tailored to a particular service area or patient group and then explaining to the clinical pharmacist why they are important ensures broad applicability to individual pharmacists in different practices and helps in setting performance expectations (in terms of clinical service quality). Such high-value actions should be incorporated into the performance management process for employees. Furthermore, focusing on high-value actions will promote the provision of standard evidence-based activities, while allowing for individualization in application of these services (depending on the patient population served) and also allowing flexibility in the overall performance management process.

Employee-level indicators used for performance management must be linked to broader organizational objectives. An example of an organizational indicator that every Canadian hospital must report is readmission rate. ${ }^{7}$ It is impossible to ascertain the direct impact of an individual pharmacist's activities on the readmission rate. However, by measuring the quantity and quality of clinical pharmacists' activities (or process 
measures) that have been shown in high-quality evidence to be linked to outcome measures such as hospital readmission, clinical coordinators or managers in the pharmacy department can rationalize why they are using those particular performance indicators to assist with performance improvement strategies for individual clinical pharmacists. For example, a Canadian study showed that patients who received team-based care incorporating a bundle of proactive clinical pharmacist activities (including high-value DTP resolution) experienced improvements in overall quality of medication use and reductions in 30-day readmission. ${ }^{8}$ It might be hypothesized that the improvement in overall quality of medication use directly led to the reductions in readmissions. Therefore, if a pharmacist resolves high-value DTPs to improve the quality of medication use, then it can logically be expected that the risk for readmission will be reduced. Performance management that incorporates evidencebased and organizationally aligned performance indicators will clarify the reasons for measuring a particular activity and assist with efforts to improve on this aspect of performance.

Applying cpKPIs to employee performance management has drawbacks. For example, if emphasis is placed solely on cpKPIs rather than considering other drivers of performance, such as emotional intelligence, improvement plans for the individual pharmacist may not be successful, because the root cause of any low performance that is exposed may not be identified. Therefore, performance indicators should be one of several tools used during the performance management process. Another caveat is the risk for indicator trade-offs, such as emphasizing productivity targets at the expense of quality. Such trade-offs can be prevented by ensuring that performance indicators are defined in the context of high-value activities before employee objectives are set.

Evidence-informed, rigorously developed cpKPIs should help to transform performance management from a largely subjective process to a more objective process in which expectations, metrics, and links to overall organizational objectives are clear to both employees and supervisors. Incorporating standardized performance indicators into the performance management process should assist in advancing clinical pharmacy practice at the employee level and should have the flexibility to help improve the quality of patient care delivered by every clinical pharmacist.

\section{References}

1. Bussières JF. Clinical pharmacy services. In: Babich M, Bornstein C, Bussières JF, Hall KW, Harding J, Lefebvre P, et al., editors. Hospital pharmacy in Canada 2009/2010 annual report. Eli Lilly; 2010 [cited 2013 Nov 13]. p. 3-20. Available from: www.lillyhospitalsurvey.ca/hpc2/content/2010_report/ 2009_2010_full_E.pdf

2. Hospital beds. In: Health at a glance 2011: OECD indicators. Paris (France): OECD Publishing; 2011.

3. DeNisi AS, Pritchard RD. Performance appraisal, performance management and improving individual performance: a motivational framework. Manage Organ Rev. 2006;2(2):253-77.

4. Armstrong M, Baron A. Performance management: the new realities. London
(UK): Institute of Personnel and Development;1998.

5. Fernandes O, Gorman S, Slavik R, Semchuk W, Doucette D, Bannerman H, et al. What are the appropriate clinical pharmacy key performance indicators for hospital pharmacists? [abstract]. Pharmacotherapy. 2013;33(10):e208.

6. Bruchet N, Loewen P, de Lemos J. Improving the quality of clinical pharmacy services: a process to identify and capture high-value "quality actions". Can J Hosp Pharm. 2011;64(1):42 7.

7. Indicators: effectiveness (quality and outcomes). In: Canadian hospital reporting project technical notes - clinical indicators. Ottawa $(\mathrm{ON})$ : Canadian Institute for Health Information;2013 [cited 2013 Nov 13]. p. 39-45. Available from: www.cihi.ca/CIHI-ext-portal/pdf/internet/CHRP_TNCI_PDF_EN

8. Makowsky MJ, Koshman SL, Midodzi WK, Tsuyuki RT. Capturing outcomes of clinical activities performed by a rounding pharmacist practicing in a team environment: the COLLABORATE study. Med Care. 2009; $47(6): 642-50$

Sean Gorman, BSc(Pharm), ACPR, PharmD

Kelowna General Hospital

Kelowna, British Columbia

Richard Slavik, PharmD, FCSHP

Interior Health Authority

Kelowna, British Columbia

Competing interests: Sean Gorman is chair of the research subgroup for the Canadian National Clinical Pharmacy Key Performance Indicator (cpKPI) Collaborative. Richard Slavik is a core member of the Canadian National Clinical Pharmacy Key Performance Indicator Collaborative

\section{THE "CON" SIDE}

A set of 8 clinical pharmacy key performance indicators (cpKPIs) for Canadian hospitals was recently established using a systematic, pan-Canadian, consensus-building (modified Delphi) process. ${ }^{1}$ A cpKPI is defined by 5 characteristics: it reflects a desired quality practice, it is a metric that is linked to direct patient care, it is associated with evidence of impact on meaningful patient outcomes, it is sensitive to pharmacist intervention, and it is feasible to measure. ${ }^{1}$ Adoption of cpKPIs may allow pharmacists to refocus and prioritize their patient care efforts on "interventions that matter" and to influence important outcomes such as readmission to hospital. Indeed, cpKPIs were developed collaboratively with the aim of advancing pharmacy practice to improve patient outcomes. ${ }^{1}$ We fully endorse the widespread implementation and measurement of cpKPIs as essential steps in advancing patient care across Canada. However, we argue that the approach of designating cpKPIs as mandatory components of individual clinical pharmacist performance assessments may actually hinder their sustained adoption. In fact, this approach may have several unintended consequences and risks, including the promotion of "bean-counting" over the needs of individual patients; of quantity over quality; of the achievement of individual pharmacists over team-based care; and of dictatorial, top-down implementation over sustained, bottom-up, inspirationally motivated practice advancement.

To begin, we would suggest that sustained, meaningful advancement of pharmacy practice is often rooted in voluntary, 
bottom-up change toward a compelling common vision. In this regard, inspirationally motivated change management involves careful consideration of stakeholders' intrinsic motivations and often trumps dictatorial approaches, with top-down "forcing functions", in terms of overall effectiveness. Including cpKPIs as mandatory components of performance appraisal may initially result in quick, transient increases for various indicators, but then may counterintuitively breed resentment and serve as a disincentive to sustained adoption among pharmacists who perceive this approach as threatening, intimidating, or an infringement on professional autonomy. Conversely, we believe that awareness of the evidence for meaningful improvement in patient outcomes from which the cpKPIs were derived ${ }^{2-5}$ will promote grassroots engagement and a sense of ownership among pharmacists, ultimately winning "hearts and minds" to advance practice and improve patient outcomes.

Second, there is a serious risk that measurement of cpKPIs may be trivialized to a point where pharmacists are "bean-counting" isolated activities to reach target thresholds rather than focusing on individual patient-specific needs. Evaluating pharmacists' performance on the basis of cpKPIs will inevitability result in disproportionate prioritization of cpKPI activities over other activities. Ultimately, patient care may suffer, if this approach results in substantial opportunity cost, whereby important non-cpKPI professional activities (such as medication safety operations, prescribing oversight, drug information, teaching, and research) become deprioritized or omitted because of a myopic "tunnel vision" ${ }^{6}$ focusing on performance appraisal designated cpKPIs. In a recent Harvard Business Review newsletter, Bregman artfully compared effective goal-setting to carefully managing prescription medications that have both efficacy benefits and unintended adverse effects.? Mandatory goal-based indicators are useful to set direction, drive behaviour, and boost performance.? However, they often unintentionally shift focus away from important nonspecific goals, fail to tap into "intrinsic motivation", and offer incentives that promote competitive "individual achievement" over team progress.?

In adopting cpKPIs, is there a risk that we will miss the forest for the trees? If cpKPIs are connected to performance appraisals, pharmacists may become unduly preoccupied with meticulously tracking activities on a checklist rather than tailoring care to each patient's individual needs. Pharmacists may subconsciously be drawn to quick wins in low-risk patients, to maximize their indicator counts, over time-consuming and resource-intensive care for complex patients who may actually need their intervention the most. An effective pharmacist adapts and responds to the individual needs of the patient population and interprofessional environment (for example, discharge patient counselling may not be a priority for critical care patients, even if it appears on the list of cpKPIs). Pharmaceutical care, which is at the heart of cpKPIs, necessitates personalized priori- tization of drug therapy problems (DTPs) and development of a care plan, rather than adoption of a fixed checklist of the same activities for all patients.

Third, if cpKPIs are used to measure individual performance, pharmacists may begin to focus on quantity over quality. For example, for the cpKPI "number of DTPs resolved", pharmacists may increase their numbers by resolving minor DTPs of little clinical significance. Increasing the number of insignificant DTPs resolved may result in a better performance evaluation without authentically improving patient care. This concept was demonstrated by Powell and others, who evaluated a system for measuring physician performance at the US Department of Veterans Affairs., They found an increase in inappropriate polypharmacy as physicians added medications to patients' regimens in an effort to meet performance targets. ${ }^{8}$

Finally, the 8 recently established cpKPIs for Canadian hospital pharmacists were designed on the basis of published papers suggesting that optimal delivery of care is a team sport. ${ }^{2,3}$ As such, the cpKPIs may reflect not the achievement of one individual pharmacist but rather the performance of a larger interprofessional and/or pharmacy team (which may include technicians and students), synergistically providing care. The randomized controlled trials of Makowsky and others ${ }^{2}$ and Gillespie and others ${ }^{3}$ highlighted significant improvements in patient outcomes when pharmacists collaborated as part of a team. Effective care was delivered as a bundle of overlapping critical elements meant to be provided together (i.e., pharmaceutical care, patient education, medication reconciliation, and active participation on rounds). These individual elements of care could be delivered by different pharmacists. It is possible that, in the early stages of implementation, some hospitals may choose to focus on just 1 or 2 cpKPI elements. This focus may lead pharmacists away from balanced care bundles that improve outcomes toward isolated cpKPIs undertaken for their contribution to individual performance appraisals. As in an orchestra, where many individual musicians contribute expertly to create harmonious music, pharmacists too must collaborate within a team to optimize patient outcomes.

Consensus-based, evidence-informed cpKPIs have enormous potential to advance hospital pharmacy practice and to improve patient outcomes at a national scale. However, sustained, effective change takes time, as we have seen with the ongoing implementation of pharmaceutical care into established practice. Indeed, timing is everything. We must first win the hearts and minds of pharmacists so they embed cpKPIs into everyday practice and then ascertain the impact of these indicators before we ponder designating cpKPIs as mandatory components of individual clinical pharmacist performance assessments.

\section{References}

1. Fernandes O, Gorman S, Slavik R, Semchuk WM, Doucette D, Bannerman $\mathrm{H}$, et al. What are the appropriate clinical pharmacy key performance indicators for hospital pharmacists? [abstract]. Pharmacotherapy. 2013;33(10):e208. 
2. Makowsky MJ, Koshman SL, Midodzi WK, Tsuyuki RT. Capturing outcomes of clinical activities performed by a rounding pharmacist practicing in a team environment: the COLLABORATE study. Med Care. 2009;47(6):642-50.

3. Gillespie U, Alassaad A, Henrohn D, Garmo H, Hammarlund-Udenaes M, Toss $\mathrm{H}$, et al. A comprehensive pharmacist intervention to reduce morbidity in patients 80 years or older. Arch Intern Med. 2009:169(9):894-990.

4. Kaboli PJ, Hoth AR, McClimon BJ, Schnipper JL. Clinical pharmacists and inpatient medical care: a systematic review. Arch Intern Med. 2006; 166(9):955-64.

5. Bond CA, Raehl CL. Clinical pharmacy services, pharmacy staffing, and hospital mortality rates. Pharmacotherapy. 2007;27(4):481-93.

6. Goddard M, Davies H, Dawson D, Mannion R, McInnes F. Clinical performance measurement: part 2-avoiding the pitfalls. J R Soc Med. 2002; 95(11):549-51.

7. Bregman P. Consider not setting goals in 2013. In: HBE blog network. Boston (MA): Harvard Business Publishing; 2012 Dec 14 [cited 2013 Nov]. Available from: http://blogs.hbr.org/bregman/2012/12/consider-not-setting-goals-in.html

8. Powell A, White K, Partin M, Halek K, Christianson J, Neil B, et al. Unintended consequences of implementing a national performance measurement system into local practice. J Gen Intern Med. 2012;27(4):405-12.

9. Kizer K, Kirsh S. The double edged sword of performance measurement. J Gen Intern Med. 2012;27(4):395-7.
Olavo A Fernandes, BScPhm, ACPR, PharmD, FCSHP Director of Pharmacy-Clinical, University Health Network Assistant Professor (Status), Leslie Dan Faculty of Pharmacy University of Toronto

Toronto, Ontario

Francesca Le Piane, BScPhm, ACPR

Staff Pharmacist, Peter Munk Cardiac Centre

Toronto General Hospital, University Health Network

Toronto, Ontario

Hina Ahmed, PharmD, BCPS

Staff Pharmacist, Cardiovascular Intensive Care

Toronto General Hospital, University Health Network

Toronto, Ontario

Kent Toombs, BSCPhm, ACPR

Clinical Pharmacy Manager, Emergency, Neurology, and Cardiology

Capital District Health Authority

Halifax, Nova Scotia

Competing interests: Olavo Fernandes and Kent Toombs serve as Co-Chairs of the Canadian National Clinical Pharmacy Key Performance Indicator (cpKPI) Collaborative and were co-investigators in the study "What Are the Appropriate National Clinical Pharmacy Key Performance Indicators for Hospital Pharmacists?" No other competing interests declared.

\section{ON THE FRONT COVER}

\section{Red Rock Coulee, Alberta}

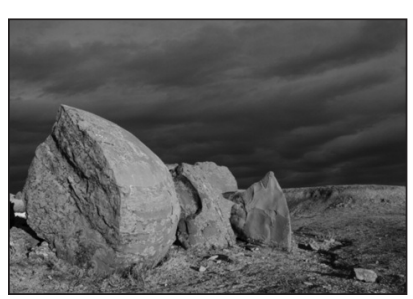

CSHP member Ken Wou (Pharmacy Manager, SouthEast, Alberta Health Services) describes this issue's cover photograph in his own words: "The prairie storm started to let up while I was driving home from Lethbridge to Medicine Hat. I decided to race to Red Rock Coulee, 15 minutes south off Highway 3, hoping to see those prehistoric stone concretions, artfully crafted by the power of nature, under the last minutes of daylight. The golden sun was setting, the black clouds were parting, and I arrived just in time. The light shone ever so blissfully on these 2.5-metre-tall reddish boulders, formed by years of deposits of sand, calcite, and iron oxide. I placed my Canon 50D (with 24-105 mm L lens) on my Gitzo tripod with a polarizing filter to capture the magnificent beauty of some of the world's largest sandstone structures among nature's angriest clouds. And in a matter of moments, the light was gone."

The CJHP would be pleased to consider photographs featuring Canadian scenery taken by CSHP members for use on the front cover of the journal. If you would like to submit a photograph, please send an electronic copy (minimum resolution $300 \mathrm{dpi}$ ) to Colleen Drake at cdrake@cshp.ca. 